Journal of Patient-Centered coAdvocateAuroraHealth

Volume 4

Issue 4 -- Health Disparities and Inequities: Part

Article 11

I

$11-6-2017$

\title{
Improved Service Efficiency Improves Racial Disparity in Diabetic Care
}

\author{
Abel Irena \\ Kushal Patel \\ David Thompson \\ Gregory Schleis \\ Abiy Gesese \\ Richard Battiola
}

Follow this and additional works at: https://aah.org/jpcrr

Part of the Clinical Epidemiology Commons, Endocrinology, Diabetes, and Metabolism Commons, Health Services Research Commons, Internal Medicine Commons, and the Nutritional and Metabolic

Diseases Commons

\section{Recommended Citation}

Irena A, Patel K, Thompson D, Schleis G, Gesese A, Battiola R. Improved service efficiency improves racial disparity in diabetic care. J Patient Cent Res Rev. 2017;4:257.

Published quarterly by Midwest-based health system Advocate Aurora Health and indexed in PubMed Central, the Journal of Patient-Centered Research and Reviews (JPCRR) is an open access, peer-reviewed medical journal focused on disseminating scholarly works devoted to improving patient-centered care practices, health outcomes, and the patient experience. 
Conclusion: Within Aurora, metabolic syndrome is rarely and variably diagnosed in medical practice, despite clear evidence of satisfied ATP III and WHO criteria.

\section{Improved Service Efficiency Improves Racial Disparity in Diabetic Care}

Abel Irena, Kushal Patel, David Thompson, Gregory Schleis, Abiy Gesese, Richard Battiola

\section{Department of Internal Medicine, Aurora Sinai Medical Center; Aurora University of Wisconsin Medical Group}

Background: Racial disparities in diabetes care have been documented. Disparities also have been shown to affect service quality and outcome of diabetic care. Analysis of our internal medicine residency clinic diabetic care management performance across REAL-G (race, ethnicity, age, preferred language and/or gender) showed race-based disparity on two outcome measures: 1) measurement of glycohemoglobin (A1C) at least twice a year; and 2) target blood pressure of $<140 / 90$.

Purpose: Develop interventions to decrease racial disparities in diabetes care among patients managed by an internal medicine residency clinic, as part of the Alliance of Independent Academic Medical Center's National Initiative V project.

Methods: Interventions were developed following analysis of clinic performance data by REAL-G categories, workflow analysis and multidisciplinary clinic team meetings. A point-of-care A1C machine was procured and workflow developed using the Plan-DoStudy-Act cycle. Staff training was conducted. A rolling 12 months data set was obtained from electronic health records. Baseline data range was December 2014 to November 2015, while endline data were from January 2016 to December 2016. The interventions were launched in July 2016. Percentage difference between baseline and endline outcome indicators was calculated and Z-score test assessed. Statistical significance was set at $\mathrm{P}<0.05$.

Results: At baseline, 62.9\% (401 of 638) of patients who selfidentified as African American/black (AA) had A1C measured at least twice a year compared to $74.3 \%$ (107 of 144) of patients who selfidentified as white/Caucasian (WC), a percentage difference of $11.4 \%$ $(\mathrm{P}=0.01)$. For goal blood pressure in diabetics, $71.0 \%$ (453 of 638 ) of AA met the target as compared to $80.6 \%$ (116 of 144) of WC, a percentage difference of $9.6 \%(\mathrm{P}=0.003)$. Following the intervention, a higher percentage of AA patients (71.4\% [381 of 534]) had at least two A1C measured during project period. The outcome also showed improvement for WC (79.8\% [95 of 119]). The percentage difference between races narrowed to $8.5 \%(\mathrm{P}=0.06)$. For goal blood pressure, $75.1 \%$ of AA achieved the target compared to $81.5 \%$ of WC, with percentage difference narrowing to $6.4 \%(\mathrm{P}=0.14)$.

Conclusion: Racial disparities in diabetes were confirmed, even for a clinic setting in which black patients are predominant. Racial disparity can be improved by implementing interventions that improve service for all patients.

\section{Generation of a Patient-Derived Brain Metastasis Breast Cancer Cell Line via Novel Orthotopic Injection Placement and Serial Mouse Transplantation to Develop PDF Mouse Model}

Amber L. LaCrosse, Denise M. Coley, Paul J. Mintz, Santhi D. Konduri, Richard A. Rovin, Amin B. Kassam
Aurora Research Institute; Aurora Neuroscience Innovation Institute

Background: The incidence of brain metastasis appears to be increasing, potentially due to advanced technology that aids early diagnosis. Patient-derived xenografts (PDX) have high translational value, as these models retain key functional characteristics of the patient tumor. PDX models are useful to understand the molecular basis of tumorigenesis and to identify new treatment targets. However, generating a first-line PDX model is challenging as engraftment failure is high. Serial transplanting tumor tissue via mouse-tomouse propagation increases engraftment rates and decreases PDX development time. Herein we report methods to generate a PDX cell line from patient-derived tumor tissue that includes the cerebral aqueduct as a novel intracranial orthotopic implantation site.

Purpose: Develop human tumors in mouse models for therapeutic purpose.

Methods: Patient-derived brain metastasis tumor tissue was enzymatically dissociated into a single cell suspension and maintained in neurocult media supplemented with human recombinant bFGF and EGF $(20 \mathrm{ng} / \mathrm{ml})$. The cells were seeded at a density of $1.0 \times 104 / \mathrm{cm}^{2}$ on ultra-low attachment plates and maintained at $37^{\circ} \mathrm{C}$ with $5 \% \mathrm{CO}_{2}$. PDX models were generated via orthotopic stereotactic surgeries. Athymic nude mice were anesthetized with an intraperitoneal injection of ketamine $(100 \mathrm{mg} / \mathrm{kg})$ and xylazine $(10 \mathrm{mg} / \mathrm{kg})$. The cerebral aqueduct was located using these coordinates from bregma: A: -5 ; L: +0.2 ; V: -2.4 . Mice were injected with $5.0 \times 104$ cells in 2 $\mu \mathrm{l}$ of media at a rate of $0.4 \mathrm{ul} / \mathrm{min}$. Mice were monitored daily for symptoms of tumor formation. Upon becoming symptomatic, mice were euthanized and tumor tissue was harvested for both culture and H\&E stain for tumor verification.

Results: Mice injected with primary patient cells (first-generation mice) developed tumors at 7 weeks (average: 6.77 weeks), secondgeneration mice yielded tumors at 2 weeks (average: 13.5 days), and third-generation mice replicated results from second-generation mice (average: 13 days). H\&E stain revealed invasive tumor masses in the ventricular system that extended from the cerebral aqueduct to the lateral ventricles. Immunohistochemistry analysis confirmed the thirdgeneration cell line retained key characteristics of the patient tumor. Conclusion: These methods successfully generated a PDX cell line from patient-derived brain metastasis that demonstrates reliable tumor formation and phenotypic stability. Importantly, our unique intracranial implantation site revealed several distinct masses, a hallmark of brain metastasis in patients.

\section{Decreasing Time to Broad Spectrum Antibiotics for Septic Patients in the Emergency Department}

Joseph J. Zieminski, Emily E. Bryant

\section{Department of Pharmacy, Aurora St. Luke's Medical Center}

Background: Timely administration of broad spectrum antibiotics has been shown to be directly correlated with decreased mortality for patients with severe sepsis and septic shock. As such, both the Surviving Sepsis Campaign and the SEP-1 CMS measure recognize timely antibiotic administration as a cornerstone of therapy for patients with severe sepsis or septic shock.

Purpose: Decrease time to broad spectrum antibiotic administration for septic patients in the emergency department (ED) of Aurora St. Luke's Medical Center.

Methods: An alert within the electronic medical record was created to more rapidly identify potentially septic patients in the ED. After 